[Chem. Pharm. Bull.

35( 3 ) $1249-1254(1987)]$

\title{
Syntheses and Properties of Dipeptides Containing $\gamma$-Aminobutyric Acid or Its Analogues at the C-Terminal ${ }^{1)}$
}

\author{
Kazuharu Ienaga, ${ }^{* a}$ Kunihiko Higashiura ${ }^{a}$ and Hiroshi Kimura ${ }^{b}$ \\ Institute of Bio-Active Science, Nippon Zoki Pharmaceutical Co., Ltd. ${ }^{a}$ \\ Kinashi, Yashiro-cho, Kato-gun, Hyogo 673-14, Japan and \\ Department of Anatomy, Shiga University of Medical \\ Science, ${ }^{b}$ Seta, Otsu 520-21, Japan
}

(Received July 14, 1986)

\begin{abstract}
Twelve dipeptides (1-12) containing $\gamma$-aminobutyric acid (GABA: $\mathrm{H}-\gamma \mathrm{Abu}-\mathrm{OH}: 37)$ at the Cterminal were synthesized. Another six dipeptides $(\mathbf{1 3}-\mathbf{1 8})$ containing GABA analogues such as $\gamma-$ amino- $\beta$-hydroxybutyric acid [GABOB: $\mathrm{H}-\gamma \mathrm{Abu}(2 \mathrm{OH})-\mathrm{OH}: 38$ ], $\beta$-alanine (39) and $\varepsilon$-aminocapric acid (EACA: $\varepsilon A c p:$ 40) at the C-terminal were also synthesized. Their properties are presented, together with preliminary findings on the immuno-crossreactivity with antiserum against GABA.
\end{abstract}

Keywords dipeptide; $\gamma$-aminobutyric acid (GABA); GABA analogue; immunohistochemistry; immuno-crossreactivity; absorption test

Many neuroscientific studies have suggested that gamma-aminobutyric acid (GABA) is a chemical neurotransmitter in the nervous system of vertebrates. ${ }^{2,3}$ Neuroanatomically, GABAergic neurons have been visualized by immunohistochemical methods, conventionally by using antisera against glutamic acid decarboxylase (an enzyme synthesizing GABA $)^{4}$ and recently by using antisera against GABA itself. ${ }^{5,6)}$ The latter method seems much better than the former, because it can directly detect the transmitter molecule. Since, however, the reliability of the method depends upon the specificity of anti-GABA serum, the immunological crossreactivity of the antiserum should be as low as possible with various other compounds that may also be present in the nervous system.

In our immunohistochemical study using an anti-GABA serum, which was proven to have very low immuno-crossreactivities with many other amino acids, ${ }^{7)}$ we noticed that some dipeptides containing GABA at the C-terminal showed quite high immunoreactivities with the anti-GABA serum. Rat brain sections, for example, that were incubated with the antiserum used as the first antibody in a usual immunohistochemical procedure, revealed numerous neuronal structures stained positively for GABA-like immunoreactivity. ${ }^{7)}$ When the antiserum had been preincubated with a crossreactive compound, the staining intensity of GABA-like immunoreactivity was decreased or even completely abolished. All the C-terminal GABA-containing dipeptides listed in Chart 1 were crossreactive on this criterion (Fig. 1). Interestingly enough, when the antiserum was absorbed with some dipeptides containing GABA at the N-terminal, such as homocarnosine, no such effect was seen.

A similar phenomenon has been reported with antisera against another amino acid, taurine. Namely, Kimura et al. ${ }^{8)}$ observed that some naturally occurring dipeptides, that contained taurine at the $\mathrm{C}$-terminal, had very high immuno-crossreactivities with antisera against taurine. Although no precise knowledge is yet available concerning the immunorecognition sites of the antigens of interest, it is very likely that many unknown dipeptides containing antigenic amino acids at the $\mathrm{C}$-terminal may also have strong crossreactivity with anti-amino acid sera. However, only a few dipeptides containing GABA at the C-terminal 
a) negative absorption
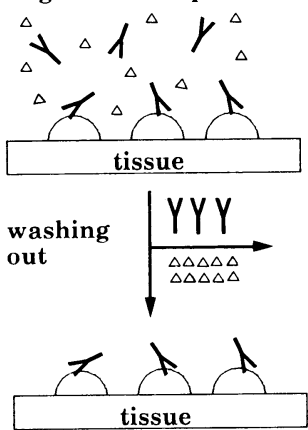

usual staining procedure

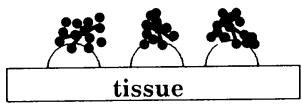

positive staining a) positive absorption
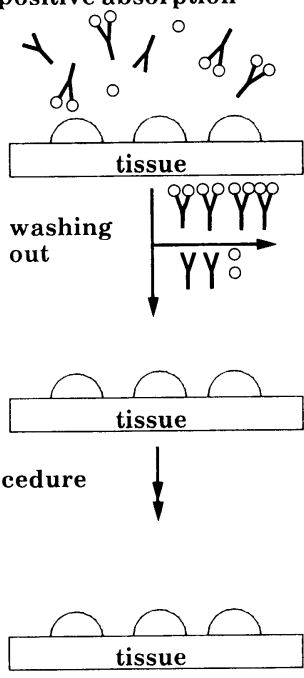

negative staining
Fig. 1. Illustration of Absorption Test for Immunohistochemistry

ـ, antigen; $>$, antibody; ::, oxidized diaminobenzidine; $\triangle$, immuno-inactive compound; $\bigcirc$, immuno-active compound.

have been synthesized.9) Further, dipeptides containing such GABA analogues as $\beta$-Ala, GABOB and EACA have not been reported so far.

In the present study, we synthesized eighteen dipeptides $(\mathbf{1}-\mathbf{1 8})$, aiming to analyze systematically their immuno-crossreactivity with GABA antiserum. We present here the details of the syntheses and some immunochemical and physiochemical properties of these unique dipeptides.

Eighteen dipeptides $(\mathbf{1}-\mathbf{1 8})$ were synthesized by the routes shown in Chart 2. Two methods using active esters were chosen for the first condensation step, and protecting groups were easily removed in a usual manner. The first method using $N$-hydroxy-5-norbornene-2,3dicarboximide (HONB) with $N, N^{\prime}$-dicyclohexylcarbodiimide (DCC) was carried out according to Fujino et al. ${ }^{10)}$ In the second method, $N, N^{\prime}$-disuccinimidyl carbonate (DSC) was used to form active esters, $\mathrm{Z}-\mathrm{X}-\mathrm{ONSu},{ }^{11)}$ and unprotected GABA or an analogue was used as the Cterminal component. Dipeptide benzyl esters containing a proline residue (Z-Pro-Y-OBzl) were difficult to purify. In contrast, Z-Pro- $\mathrm{Y}-\mathrm{OH}$ was easily obtained as a pure crystalline

$\mathrm{H}-\mathrm{X}-\gamma \mathrm{Abu}-\mathrm{OH}$

X: Gly (1) Ala (2) Val (3) Leu (4) Ile (5) Pro (6) Hyp (7)

Ser (8) Thr (9) Phe (10) Tyr (11) Asp (12)

$\mathrm{H}-\mathrm{X}-\gamma \mathrm{Abu}(2 \mathrm{OH})-\mathrm{OH} \quad \mathrm{H}-\mathrm{X}-\beta \mathrm{Ala}-\mathrm{OH} \quad \mathrm{H}-\mathrm{X}-\varepsilon \mathrm{Acp}-\mathrm{OH}$

$X$ : Phe (13) Pro (14) $X$ : Phe (15) Pro (16) $X:$ Phe (17) Pro (18)

$\mathrm{Z}-\mathrm{X}-\gamma \mathrm{Abu}-\mathrm{OBzl}$

$X$ : Gly (19) Ala (20) Val (21) Leu (22) Ile (23) Hyp (24)

Ser (25) Thr (26) Phe (27) Tyr(OBzl) (28) Asp(OBzl) (29)

$\mathrm{Z}-\mathrm{X}-\gamma \mathrm{Abu}-\mathrm{OH} \quad \mathrm{Z}-\mathrm{X}-\gamma \mathrm{Abu}(2 \mathrm{OH})-\mathrm{OBzl} \quad \mathrm{Z}-\mathrm{X}-\gamma \mathrm{Abu}(2 \mathrm{OH})-\mathrm{OH}$

$\mathrm{X}$ : Pro $(30) \quad \mathrm{X}$ : Phe $(31) \quad X$ : Pro (32)

Z-Phe- $\beta$ Ala-OH Z-Pro- $\varepsilon$ Acp-OH

$X$ : Phe (33) Pro (34) $X$ : Phe (35) Pro (36)

$\mathrm{H}-\gamma \mathrm{Abu}-\mathrm{OH}(37) \quad \mathrm{H}-\gamma \mathrm{Abu}(2 \mathrm{OH})-\mathrm{OH}(38) \quad \mathrm{H}-\beta \mathrm{Ala}-\mathrm{OH}(39) \quad \mathrm{H}-\varepsilon \mathrm{Acp}-\mathrm{OH}(40)$ 
method A

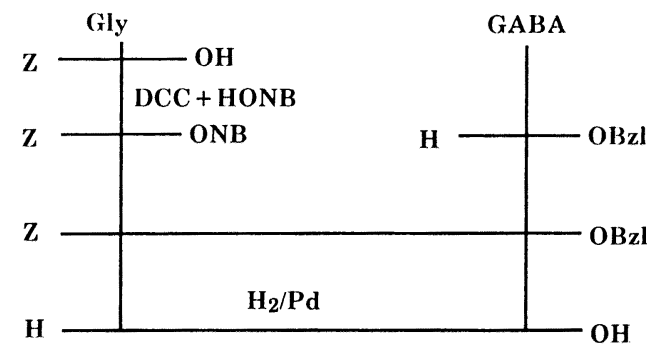

method B

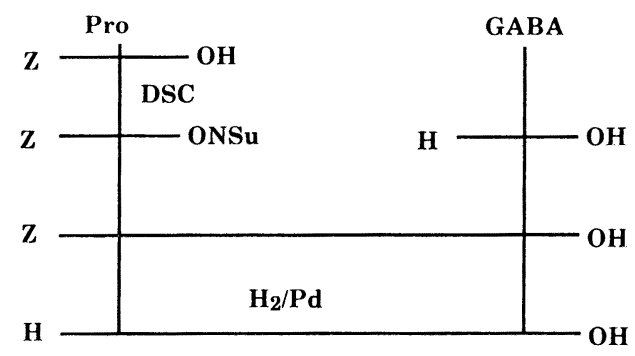

Chart 2

TABlE I. Specific Optical Rotation Values at the Sodium D Line of Neutral Dipeptides and Their Amino Acid Components ${ }^{a)}$ at $20-25^{\circ} \mathrm{C}$

\begin{tabular}{|c|c|c|c|}
\hline \multirow{2}{*}{ Compd. } & \multirow{2}{*}{$\begin{array}{c}\text { Dipeptides }(\mathrm{H}-\mathrm{X}-\mathrm{Y}-\mathrm{OH}) \\
{[\alpha]_{\mathrm{D}}\left(c=1, \mathrm{H}_{2} \mathrm{O}\right)}\end{array}$} & \multicolumn{2}{|c|}{ Amino acid components $(\mathrm{H}-\mathrm{X}-\mathrm{OH})$} \\
\hline & & {$[\alpha]_{\mathrm{D}}\left(c=1-5, \mathrm{H}_{2} \mathrm{O}\right)$} & {$[\alpha]_{\mathrm{D}}(c=1-5$, aq. $\mathrm{HCl})$} \\
\hline 2 & $+15.1^{\circ}$ & +1.8 & $+14.6^{\circ}$ \\
\hline 3 & +40.7 & +5.6 & $+28.3^{\circ}$ \\
\hline 4 & +31.6 & -11.0 & $+28.3^{\circ}$ \\
\hline 5 & +28.6 & +12.4 & $+39.5^{\circ}$ \\
\hline 6 & -47.3 & -86.2 & -60.4 \\
\hline 14 & -31.9 & $-86.2^{\circ}$ & -60.4 \\
\hline 16 & $-50.8^{\circ}$ & $-86.2^{\circ}$ & $-60.4^{\circ}$ \\
\hline 18 & -47.6 & -86.2 & $-60.4^{\circ}$ \\
\hline 7 & -35.8 & $-76.0^{\circ}$ & $-50.5^{\circ}$ \\
\hline 8 & $+12.5^{\circ}$ & $-7.5^{\circ}$ & $+15.1^{\circ}$ \\
\hline 9 & $+19.2^{\circ}$ & $-28.5^{\circ}$ & $-15.0^{\circ}$ \\
\hline 10 & $+54.1^{\circ}$ & $-34.5^{\circ}$ & $-4.5^{\circ}$ \\
\hline 13 & $+60.3^{\circ}$ & $-34.5^{\circ}$ & $-4.5^{\circ}$ \\
\hline 15 & $+63.4^{\circ}$ & $-34.5^{\circ}$ & $-4.5^{\circ}$ \\
\hline 17 & $+42.9^{\circ}$ & $-34.5^{\circ}$ & $-4.5^{\circ}$ \\
\hline 11 & $+44.6^{\circ}$ & b) & $-10.5^{\circ}$ \\
\hline
\end{tabular}

a) "Data for Biochemical Research," ed. by R. M. C. Dawson, D. Eliott, W. H. Eliott and K. M. Jones, Clarendon Press, Oxford, 1974. b) Insoluble.

solid, so that dipeptides containing proline were synthesized by the latter method.

Specific optical rotation values of sixteen neutral dipeptides (2-18) and their amino acid components are shown in Table I. The $[\alpha]_{D}$ values of the dipeptides, except Ser, Phe and Tyr derivatives $(8,10,11,13,15,17)$, were similar to those of the amino acids located at the N- 
TABLE II. Proton Magnetic Resonance Spectra of Dipeptides $(\mathrm{H}-\mathrm{X}-\mathrm{Y}-\mathrm{OH})$ and C-Terminal Amino Acids $(\mathrm{H}-\mathrm{Y}-\mathrm{OH})$

\begin{tabular}{|c|c|c|}
\hline & Signals of N-terminal moiety $\left(-\mathrm{X}_{-}\right)$ & Signals of C-terminal moiety $(-\mathrm{Y}-)$ \\
\hline 1 & $3.77(2 \mathrm{H}, \mathrm{s})$ & $\begin{array}{l}1.77(\mathrm{tt}, J=7.5,7, \beta \mathrm{H}), 2.25(\mathrm{t}, J=7.5, \alpha \mathrm{H}), \\
3.25(\mathrm{t}, J=7, \gamma \mathrm{H})\end{array}$ \\
\hline 2 & $1.50(3 \mathrm{H}, \mathrm{d}, J=7), 4.02(1 \mathrm{H}, \mathrm{q}, J=7)$ & $\begin{array}{l}1.78(\mathrm{tt}, J=7,7, \beta \mathrm{H}), 2.29(\mathrm{t}, J=7, \alpha \mathrm{H}), 3.24 \\
3.27(\mathrm{dt}, J=7,14, \gamma \mathrm{H})\end{array}$ \\
\hline 3 & $\begin{array}{l}1.01(3 \mathrm{H}, \mathrm{d}, J=7), 1.02(3 \mathrm{H}, \mathrm{d}, J=7), 2.12- \\
2.24(1 \mathrm{H}, \mathrm{m}), 3.72(1 \mathrm{H}, \mathrm{d}, J=7)\end{array}$ & $\begin{array}{l}1.80(\mathrm{tt}, J=7,7, \beta \mathrm{H}), 2.31(\mathrm{t}, J=7, \alpha \mathrm{H}), 3.24 \\
3.30(\mathrm{dt}, J=7,14, \gamma \mathrm{H})\end{array}$ \\
\hline 4 & $\begin{array}{l}0.94(3 \mathrm{H}, \mathrm{d}, J=6), 0.96(3 \mathrm{H}, \mathrm{d}, J=6), 1.58 \\
1.75(3 \mathrm{H}, \mathrm{m}), 3.94(1 \mathrm{H}, \mathrm{t}, J=7)\end{array}$ & $\begin{array}{l}1.81(\mathrm{tt}, J=7,7, \beta \mathrm{H}), 2.36(\mathrm{t}, J=7, \alpha \mathrm{H}), 3.22 \\
3.32(\mathrm{dt}, J=7,14, \gamma \mathrm{H})\end{array}$ \\
\hline 5 & $\begin{array}{l}0.92(3 \mathrm{H}, \mathrm{t}, J=7), 0.98(3 \mathrm{H}, \mathrm{d}, J=7), 1.17- \\
1.29(1 \mathrm{H}, \mathrm{m}), 1.45-1.56(1 \mathrm{H}, \mathrm{m}), 1.89-2.00 \\
(1 \mathrm{H}, \mathrm{m}), 3.79(1 \mathrm{H}, \mathrm{t}, J=6)\end{array}$ & $\begin{array}{l}1.80(\mathrm{tt}, J=7,7, \beta \mathrm{H}), 2.34(\mathrm{t}, J=7, \alpha \mathrm{H}), 3.23 \\
3.31(\mathrm{dt}, J=7,14, \gamma \mathrm{H})\end{array}$ \\
\hline 6 & $\begin{array}{l}1.97-2.11(3 \mathrm{H}, \mathrm{m}), 2.36-2.49(1 \mathrm{H}, \mathrm{m}), 3.34- \\
3.77(2 \mathrm{H}, \mathrm{m}), 4.32(1 \mathrm{H}, \mathrm{dd}, J=7,8)\end{array}$ & $\begin{array}{l}1.79(\mathrm{tt}, J=7,7, \beta \mathrm{H}), 2.29(\mathrm{t}, J=7, \alpha \mathrm{H}), 3.24 \\
3.29(\mathrm{dt}, J=7,14, \gamma \mathrm{H})\end{array}$ \\
\hline 7 & $\begin{array}{l}2.15(1 \mathrm{H}, \text { ddd, } J=3.5,10,14), 2.47(1 \mathrm{H}, \text { dddd } \\
J=2,2,8,14), 3.41(1 \mathrm{H}, \mathrm{ddd}, J=2,2,12.5) \\
4.53(1 \mathrm{H}, \mathrm{dd}, J=8,10), 4.68-4.72(1 \mathrm{H}, \mathrm{m})\end{array}$ & $\begin{array}{l}1.80(\mathrm{tt}, J=7,7, \beta \mathrm{H}), 2.33(\mathrm{t}, J=7, \alpha \mathrm{H}), 3.25 \\
3.31(\mathrm{dt}, J=7,14, \gamma \mathrm{H})\end{array}$ \\
\hline 8 & $\begin{array}{l}3.91(1 \mathrm{H}, \mathrm{dd}, J=6,12.5), 3.97(1 \mathrm{H}, \mathrm{dd}, J=4 \\
12.5), 4.07(1 \mathrm{H}, \mathrm{dd}, J=4,6)\end{array}$ & $\begin{array}{l}1.79(\mathrm{tt}, J=7.5,7.5, \beta \mathrm{H}), 2.29(\mathrm{t}, J=7,5, \alpha \mathrm{H}), \\
3.27(\mathrm{t}, J=7.5, \gamma \mathrm{H})\end{array}$ \\
\hline 9 & $\begin{array}{l}1.29(3 \mathrm{H}, \mathrm{d}, J=6.5), 3.79(1 \mathrm{H}, \mathrm{d}, J=6), 4.12 \\
(1 \mathrm{H}, \mathrm{dq}, J=6,6.5)\end{array}$ & $\begin{array}{l}1.81(\mathrm{tt}, J=7,7, \beta \mathrm{H}), 2.37(\mathrm{t}, J=7, \alpha \mathrm{H}), 3.28 \\
(\mathrm{t}, J=7, \gamma \mathrm{H})\end{array}$ \\
\hline 10 & $\begin{array}{l}3.09(1 \mathrm{H}, \mathrm{dd}, J=9,13), 3.23(1 \mathrm{H}, \mathrm{dd}, J=6,13) \\
4.12(1 \mathrm{H}, \mathrm{dd}, J=6,9), 7.24-7.42(5 \mathrm{H}, \mathrm{m})\end{array}$ & $\begin{array}{l}1.46-1.63(\mathrm{~m}, \beta \mathrm{H}), 1.94-2.11(\mathrm{~m}, \alpha \mathrm{H}), 2.98 \\
3.25(\mathrm{dt}, J=7,14, \gamma \mathrm{H})\end{array}$ \\
\hline 11 & $\begin{array}{l}3.00(1 \mathrm{H}, \mathrm{dd}, J=9,14), 3.16(1 \mathrm{H}, \mathrm{dd}, J=6,14) \\
4.06(1 \mathrm{H}, \mathrm{dd}, J=6,9), 6.86(2 \mathrm{H}, \mathrm{d}, J=8), 7.13 \\
(2 \mathrm{H}, \mathrm{d}, J=8)\end{array}$ & $\begin{array}{l}1.48-1.61(\mathrm{~m}, \beta \mathrm{H}), 1.97-2.13(\mathrm{~m}, \alpha \mathrm{H}), 2.97 \\
3.29(\mathrm{dt}, J=7,14, \gamma \mathrm{H})\end{array}$ \\
\hline 12 & $\begin{array}{l}2.95(1 \mathrm{H}, \mathrm{dd}, J=7,18), 3.01(1 \mathrm{H}, \mathrm{dd}, J=6,18) \\
4.27(1 \mathrm{H}, \mathrm{dd}, J=6,7)\end{array}$ & $\begin{array}{l}1.81(\mathrm{tt}, J=7,7, \beta \mathrm{H}), 2.40(\mathrm{t}, J=7, \alpha \mathrm{H}), 3.23 \\
3.33(\mathrm{dt}, J=7,13.5, \gamma \mathrm{H})\end{array}$ \\
\hline 37 & & $\begin{array}{l}1.90(\mathrm{tt}, J=7.5,7.5, \beta \mathrm{H}), 2.34(\mathrm{t}, J=7.5, \alpha \mathrm{H}) \\
3.01(\mathrm{t}, J=7.5, \gamma \mathrm{H})\end{array}$ \\
\hline 13 & $\begin{array}{l}3.10-3.29(2 \mathrm{H}, \mathrm{m}), 4.17-4.23(1 \mathrm{H}, \mathrm{m}), 7.25- \\
7.45(5 \mathrm{H}, \mathrm{m})\end{array}$ & $\begin{array}{l}2.09-2.32(\mathrm{~m}, \alpha \mathrm{H}), 3.10-3.29(\mathrm{~m}, \gamma \mathrm{H}), 3.88- \\
4.35(\mathrm{~m}, \beta \mathrm{H})\end{array}$ \\
\hline 14 & $\begin{array}{l}2.01-2.11(3 \mathrm{H}, \mathrm{m}), 2.41-2.54(1 \mathrm{H}, \mathrm{m}), 3.30- \\
3.48(2 \mathrm{H}, \mathrm{m}), 4.37(1 \mathrm{H}, \mathrm{dd}, J=7,8)\end{array}$ & $\begin{array}{l}2.41-2.54,2.58-2.65(\mathrm{~m}, \alpha \mathrm{H}), 3.30-3.48(\mathrm{~m}, \\
\gamma \mathrm{H}), 4.13-4.22(\mathrm{~m}, \beta \mathrm{H})\end{array}$ \\
\hline 38 & & $\begin{array}{l}2.46(\mathrm{dd}, J=7,15, \alpha \mathrm{H}), 2.51(\mathrm{dd}, J=5,15, \alpha \mathrm{H}) \\
2.96(\mathrm{dd}, J=9,13, \gamma \mathrm{H}), 3.17(\mathrm{dd}, J=3,13, \gamma \mathrm{H}), \\
4.19-4.26(\mathrm{~m}, \beta \mathrm{H})\end{array}$ \\
\hline 15 & $\begin{array}{l}3.10(1 \mathrm{H}, \mathrm{dd}, J=9,14), 3.21(1 \mathrm{H}, \mathrm{dd}, J=6,14) \\
4.15(1 \mathrm{H}, \mathrm{dd}, J=6,9), 7.20-7.43(5 \mathrm{H}, \mathrm{m})\end{array}$ & $\begin{array}{l}2.34(\mathrm{ddd}, J=5,8,17, \alpha \mathrm{H}), 2.44(\mathrm{ddd}, J=5,6 \\
17, \alpha \mathrm{H}), 3.25(\mathrm{ddd}, J=5,8,14, \beta \mathrm{H}), 3.45(\mathrm{ddd} \\
J=5,6,14, \beta \mathrm{H})\end{array}$ \\
\hline 16 & $\begin{array}{l}2.10-2.18(3 \mathrm{H}, \mathrm{m}), 2.38-2.47(1 \mathrm{H}, \mathrm{m}), 3.35- \\
3.46(2 \mathrm{H}, \mathrm{m}), 4.35(1 \mathrm{H}, \mathrm{dd}, J=7,8)\end{array}$ & $\begin{array}{l}2.64(\mathrm{t}, J=6.5, \alpha \mathrm{H}), 3.49,3.57(\mathrm{dd}, J=6.5,13 \\
\beta \mathrm{H})\end{array}$ \\
\hline 39 & & $2.58(\mathrm{t}, J=6.5, \alpha \mathrm{H}), 3.18(\mathrm{t}, J=6.5, \beta \mathrm{H})$ \\
\hline 17 & $\begin{array}{l}3.08(1 \mathrm{H}, \mathrm{dd}, J=9,13), 3.22(1 \mathrm{H}, \mathrm{dd}, J=6,13) \\
4.12(1 \mathrm{H}, \mathrm{dd}, J=6,9), 7.20-7.45(5 \mathrm{H}, \mathrm{m})\end{array}$ & $\begin{array}{l}0.97-1.07(\mathrm{~m}, \gamma \mathrm{H}), 1.22-1.34(\mathrm{~m}, \delta \mathrm{H}), 1.48 \\
(\mathrm{tt}, J=7,7, \beta \mathrm{H}), 2.30(\mathrm{t}, J=7, \alpha \mathrm{H}), 2.91-3.00 \\
3.15-3.23(\mathrm{~m}, \varepsilon \mathrm{H})\end{array}$ \\
\hline 18 & $\begin{array}{l}1.96-2.11(3 \mathrm{H}, \mathrm{m}), 2.39-2.49(1 \mathrm{H}, \mathrm{m}), 3.35- \\
3.48(2 \mathrm{H}, \mathrm{m}), 4.33(1 \mathrm{H}, \mathrm{t}, J=7.5)\end{array}$ & $\begin{array}{l}1.30-1.38(\mathrm{~m}, \gamma \mathrm{H}), 1.49-1.60(\mathrm{~m}, \delta \mathrm{H}), 1.60 \\
(\mathrm{tt}, J=8,8, \beta \mathrm{H}), 2.38(\mathrm{t}, J=8, \alpha \mathrm{H}), 3.19,3.29 \\
(\mathrm{dt}, J=7,14, \varepsilon \mathrm{H})\end{array}$ \\
\hline 40 & & $\begin{array}{l}1.29-1.38(\mathrm{~m}, \gamma \mathrm{H}), 1.55(\mathrm{tt}, J=7.5,7.5, \delta \mathrm{H}) \\
1.62(\mathrm{tt}, J=7.5,7.5, \beta \mathrm{H}), 2.20(\mathrm{t}, J=7.5, \alpha \mathrm{H}) \\
2.24(\mathrm{t}, J=7.5, \varepsilon \mathrm{H})\end{array}$ \\
\hline
\end{tabular}


terminal under acidic conditions. However, no relationship was apparent between the values of the dipeptides and those of the amino acids under neutral conditions (around $\mathrm{pH} 7$ ). The values seemed to depend not on the total charge of molecules, but on the charge of the hetero atom next to the carbonyl group: $\mathrm{CO}-\underline{\mathrm{NH}}-\mathrm{CO}-\underline{\mathrm{OH}}$ or $\mathrm{CO}-\underline{\mathrm{O}}^{-}$.

Table II shows the $\delta$ and $J$ values of dipeptides $(\mathbf{1}-\mathbf{1 8})$ in $0.1 \mathrm{~N} \mathrm{DCl}$, taken from the proton nuclear magnetic resonance $\left({ }^{1} \mathrm{H}-\mathrm{NMR}\right)$ spectra. Signals derived from GABA were characteristic. The chemical shifts of $\alpha \mathrm{H}, \beta \mathrm{H}$ and $\gamma \mathrm{H}$ of the GABA residue in these peptides were similar to those of GABA itself.

The peptide containing Gly (1) had one kind of $\gamma \mathrm{H}$ proton signal, while most of the other peptides $(2-7,10-12)$ had two kinds of $\gamma \mathrm{H}$ proton signals. Two other peptides $(\mathbf{8}, \mathbf{9})$, however, containing Ser and Thr, respectively, also showed only one kind of $\gamma \mathrm{H}$ proton signal.

All of the dipeptides studied in this paper possessed more or less strong immunological absorption capability as mentioned above. A quantitative evaluation of the immunocrossreactivity of the anti-GABA serum is in progress.

\section{Experimental}

Melting points of all compounds are uncorrected. ${ }^{1} \mathrm{H}-\mathrm{NMR}$ spectra were obtained by using tert-butanol $(1.23 \mathrm{ppm})$ as an internal standard with a Brucker AM-400 spectrometer. Optical rotations were measured with a JASCO DIP-140 spectrometer.

Immunostaining Procedure - The avidin-biotin-complex ( $\mathrm{ABC}$ ) method $^{12)}$ was used as an immunohistochemical procedure. Immunostaining for GABA-like immunoreactivity was carried out in rat brain tissues according to the procedure described by Kimura and Tanaka. ${ }^{6}$ For absorption tests, only the first step of the staining procedure was modified. Namely, a $0.3 \%$ Triton-X solution $(100 \mu \mathrm{l})$, in which a test sample $(3.3 \mathrm{mg}$ or none) was dissolved, was added to a diluted solution of anti-GABA serum $(1 \mathrm{ml} ; 1: 10000)$. The absorption ability of the sample was judged from the staining intensity compared with that of the non-absorbed control.

Method A-a) Z-Hyp- $-\mathrm{Abu}-\mathrm{OBzl}$ (24): DCC (4.54 g) was added to a solution of Z-Hyp-OH (4.78 g) and HONB $(4.34 \mathrm{~g})$ in THF $(100 \mathrm{ml})$ at $0{ }^{\circ} \mathrm{C}$, and the mixture was stirred at the same temperature for an additional $1 \mathrm{~h}$, then at room temperature for $1 \mathrm{~h}$. Z-Hyp-ONB was formed, and the resulting DCU was filtered off. The filtrate was concentrated in vacuo. The oily residue was dissolved in dioxane $(100 \mathrm{ml})$, and to this solution, GABA-OBzl $p$ toluene sulfonate $(7.31 \mathrm{~g})$ and triethylamine $(2.02 \mathrm{~g})$ were added at room temperature. After stirring of the mixture for $20 \mathrm{~h}$ followed by evaporation in vacuo, the oily residue was dissolved in ethyl acetate $(100 \mathrm{ml})$. The solution was washed successively with water, $5 \%$ aqueous sodium hydrogen carbonate, brine, $10 \%$ aqueous citric acid and brine. After being dried over sodium sulfate, the organic layer was evaporated to dryness in vacuo. The crystalline residue was recrystallized from ethyl acetate-ether $(15 \mathrm{ml}: 50 \mathrm{ml})$ to yield pure Z-Hyp- $\gamma \mathrm{Abu}-\mathrm{OBzl}(\mathbf{2 4})(7.15 \mathrm{~g}, 81 \%), \mathrm{mp} 72-$ $73^{\circ} \mathrm{C},[\alpha]_{\mathrm{D}}-15.0^{\circ}(c=1, \mathrm{DMF})$.

Eleven other protected dipeptides (19-23, 25-30) were similarly synthesized: Z-Gly- $\gamma \mathrm{Abu}-\mathrm{OBzl}(\mathbf{1 9})(84 \%)$, mp 83.5-85 ${ }^{\circ}$; Z-Ala- $\gamma$ Abu-OBzl (20) $(88 \%)$, mp 99.5-100.5 ${ }^{\circ} \mathrm{C},[\alpha]_{\mathrm{D}}+6.4^{\circ}(c=1$, DMF); Z-Val- $\gamma$ Abu-OBzl (21) $(78 \%), \mathrm{mp} 114.5-116^{\circ} \mathrm{C},[\alpha]_{\mathrm{D}}+10.1^{\circ}\left(c=1\right.$, DMF); Z-Leu- $\gamma$ Abu-OBzl (22) $(50 \%), \mathrm{mp} 73-74^{\circ} \mathrm{C},[\alpha]_{\mathrm{D}}-3.8^{\circ}$ $\left(c=1\right.$, DMF); Z-Ile- $\gamma$ Abu-OBzl (23) $\left(56^{\circ}\right), \mathrm{mp} 106.5-109^{\circ} \mathrm{C},[\alpha]_{\mathrm{D}}+6.7^{\circ}(c=1, \mathrm{DMF}) ; \mathrm{Z}-\mathrm{Ser}-\gamma \mathrm{Abu}-\mathrm{OBzl}(\mathbf{2 5})$ $\left(61^{\circ}\right), \mathrm{mp} 137-138^{\circ} \mathrm{C},[\alpha]_{\mathrm{D}}+17.5^{\circ}(c=1, \mathrm{DMF}) ; \mathrm{Z}-\mathrm{Thr}-\gamma \mathrm{Abu}-\mathrm{OBzl}(\mathbf{2 6})\left(95^{\circ}\right), \mathrm{mp} 94.5-95.5^{\circ} \mathrm{C},[\alpha]_{\mathrm{D}}+2.0^{\circ}$ $\left(c=1\right.$, DMF); Z-Phe- $\gamma$ Abu-OBzl (27) $(70 \%)$, mp 133-134 ${ }^{\circ} \mathrm{C},[\alpha]_{\mathrm{D}}-8.9^{\circ}(c=1, \mathrm{DMF}) ; \mathrm{Z}-\mathrm{Tyr}(\mathrm{OBzl})-\gamma \mathrm{Abu}-\mathrm{OBzl}$ (28) $\left(92^{\circ} \%\right), \mathrm{mp} 138-139^{\circ} \mathrm{C},[\alpha]_{\mathrm{D}}-13.0^{\circ}(c=1, \mathrm{DMF}) ; \mathrm{Z}-\mathrm{Asp}(\mathrm{OBzl})-\gamma \mathrm{Abu}-\mathrm{OBzl}(29)\left(87^{\circ} \%\right), \mathrm{mp} 111-112^{\circ} \mathrm{C},[\alpha]_{\mathrm{D}}$ $-6.5^{\circ}(c=1, \mathrm{DMF})$; Z-Phe- $\gamma$ Abu $(2 \mathrm{OH})-\mathrm{OBzl}(32)(70 \%), \mathrm{mp} 122-123^{\circ} \mathrm{C},[\alpha]_{\mathrm{D}}-10.8^{\circ}(c=1$, DMF $)$.

b) $\mathrm{H}-\mathrm{Gly}-\gamma \mathrm{Abu}-\mathrm{OH}$ (1): Hydrogenation of Z-Gly- $\gamma \mathrm{Abu}-\mathrm{OBzl}$ (19) (4.6 g) over a Pd catalyst in the usual manner in a mixed solvent of $\mathrm{MeOH}-\mathrm{AcOH}-\mathrm{H}_{2} \mathrm{O}(30 \mathrm{ml}: 10 \mathrm{ml}: 5 \mathrm{ml})$, followed by removal of the catalyst and evaporation of the solvent, gave a residue. The residue was dissolved in a mixed solvent of $\mathrm{EtOH}$ and toluene $(20 \mathrm{ml}: 30 \mathrm{ml})$, and the solution was evaporated to dryness to give a colorless solid. Recrystallization from $\mathrm{H}_{2} \mathrm{O}-\mathrm{EtOH}$ gave pure $\mathrm{H}-\mathrm{Gly}-$ $\gamma \mathrm{Abu}-\mathrm{OH}(1),\left(2.05 \mathrm{~g}, 85^{\circ}\right.$ ) , mp 212-215 ${ }^{\circ} \mathrm{C}$ (dec.). Anal. Calcd for $\mathrm{C}_{6} \mathrm{H}_{12} \mathrm{~N}_{2} \mathrm{O}_{3}: \mathrm{C}, 44.99 ; \mathrm{H}, 7.55 ; \mathrm{N}, 17.49$. Found: C, 44.97; H, 7.62; N, 17.41 .

Ten other dipeptides $(\mathbf{2}-\mathbf{1 1}, \mathbf{1 3})$ were similarly obtained: $\mathrm{H}-\mathrm{Ala}-\gamma \mathrm{Abu}-\mathrm{OH}(\mathbf{2})\left(87^{\circ}\right), \mathrm{mp} 215-218^{\circ} \mathrm{C}(\mathrm{dec}$.). Anal. Calcd for $\mathrm{C}_{7} \mathrm{H}_{14} \mathrm{~N}_{2} \mathrm{O}_{3}: \mathrm{C}, 48.26 ; \mathrm{H}, 8.10 ; \mathrm{N}, 16.08$. Found: C, 48.26; H, 8.04; N, 15.98; H-Val- $\gamma \mathrm{Abu}-\mathrm{OH}$ (3) $\left(69^{\circ}\right)$, mp 202-203 ${ }^{\circ} \mathrm{C}$. Anal. Calcd for $\mathrm{C}_{9} \mathrm{H}_{18} \mathrm{~N}_{2} \mathrm{O}_{3}: \mathrm{C}, 53.44 ; \mathrm{H}, 8.97 ; \mathrm{N}, 13.85$. Found: C, 53.78; H, 9.02; N, 14.00; H-Leu- - Abu-OH (4) $(73 \%)$, mp 179.5-180.5 C. Anal. Calcd for $\mathrm{C}_{10} \mathrm{H}_{20} \mathrm{~N}_{2} \mathrm{O}_{3}: \mathrm{C}, 55.55 ; \mathrm{H}, 9.47 ; \mathrm{N}, 13.07$. Found: $\mathrm{C}, 55.53 ; \mathrm{H}, 9.32 ; \mathrm{N}, 12.95 ; \mathrm{H}-\mathrm{Ile}-\gamma \mathrm{Abu}-\mathrm{OH}(5)(56 \%), \mathrm{mp} 152-154^{\circ} \mathrm{C}$ (amorphous); H-Hyp- $\gamma \mathrm{Abu}-\mathrm{OH}(7)(75 \%)$, 
mp 210-211 C. Anal. Calcd for $\mathrm{C}_{9} \mathrm{H}_{16} \mathrm{~N}_{2} \mathrm{O}_{4}: \mathrm{C}, 49.99 ; \mathrm{H}, 7.46 ; \mathrm{N}, 12.96$. Found: C, 49.85; H, 7.87; N, 12.66; H-Ser$\gamma \mathrm{Abu}-\mathrm{OH}(8)(82 \%)$, mp $188-191{ }^{\circ} \mathrm{C}$ (dec.). Anal. Calcd for $\mathrm{C}_{7} \mathrm{H}_{14} \mathrm{~N}_{2} \mathrm{O}_{4}: \mathrm{C}, 44.20 ; \mathrm{H}, 7.42 ; \mathrm{N}, 14.73$. Found: C,

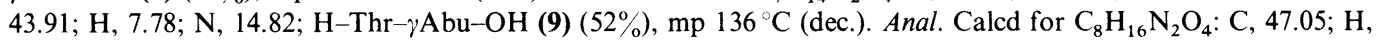
7.90; N, 13.72. Found: C, 47.25; H, 7.99; N, 13.89; H-Phe- $\gamma \mathrm{Abu}-\mathrm{OH}(10)(82 \%), \mathrm{mp} 173.5-174.5^{\circ} \mathrm{C}$, Anal. Calcd for $\mathrm{C}_{13} \mathrm{H}_{18} \mathrm{~N}_{2} \mathrm{O}_{3}: \mathrm{C}, 62.38 ; \mathrm{H}, 7.25 ; \mathrm{N}, 11.19$. Found: $\mathrm{C}, 62.03 ; \mathrm{H}, 7.38 ; \mathrm{N}, 10.95 ; \mathrm{H}-\mathrm{Tyr}-\gamma \mathrm{Abu}-\mathrm{OH}(11)(72 \%), \mathrm{mp}$ $68-71{ }^{\circ} \mathrm{C}$ (amorphous). $\mathrm{H}-\mathrm{Asp}-\gamma \mathrm{Abu}-\mathrm{OH}(12)(83 \%), \operatorname{mp} 216-218^{\circ} \mathrm{C}\left(\mathrm{dec}\right.$.), $[\alpha]_{\mathrm{D}}+12.6^{\circ}(c=1,0.1 \mathrm{~N} \mathrm{NaOH})$. Anal. Calcd for $\mathrm{C}_{8} \mathrm{H}_{14} \mathrm{~N}_{2} \mathrm{O}_{5}: \mathrm{C}, 44.03 ; \mathrm{H}, 6.47 ; \mathrm{N}, 12.84$. Found: C, 44.00; H, 6.61; N, 12.59; H-Phe- $\gamma \mathrm{Abu}(2 \mathrm{OH})-\mathrm{OH}(13)$ $(75 \%), \mathrm{mp} 177-179{ }^{\circ} \mathrm{C}$. Anal. Calcd for $\mathrm{C}_{13} \mathrm{H}_{18} \mathrm{~N}_{2} \mathrm{O}_{4}: \mathrm{C}, 58.63 ; \mathrm{H}, 6.81 ; \mathrm{N}, 10.52$. Found: C, 58.41; H, 6.90; N, 10.42 .

Method B-a) Z-Pro- $\gamma$ Abu-OH (31): Z-Pro-ONSu $(1.73 \mathrm{~g})$ in acetonitrile $(30 \mathrm{ml})$ was added to a solution of $\operatorname{GABA}(0.52 \mathrm{~g})$ and TEA $(0.51 \mathrm{~g})$ in water-acetonitrile $(10 \mathrm{ml}: 10 \mathrm{ml})$ at room temperature. After stirring of the mixture for $20 \mathrm{~h}$ and evaporation in vacuo, the residue was dissolved in a mixed solvent of $1 \mathrm{~N} \mathrm{aq.} \mathrm{HCl}(20 \mathrm{ml})$ and ethyl acetate $(50 \mathrm{ml})$. The organic layer was separated, washed with brine and extracted with $5 \%$ aqueous sodium hydrogen carbonate $(30 \mathrm{ml} \times 3)$. The extract was washed with ethyl acetate $(30 \mathrm{ml} \times 2)$ and acidified with $6 \mathrm{~N}$ aq. $\mathrm{HCl}$. The separated oil was extracted with ethyl acetate $(30 \mathrm{ml} \times 3)$ and the organic solution was washed with brine $(30 \mathrm{ml})$, dried over sodium sulfate and evaporated in vacuo. The oily residue was triturated with petroleum ether. The crystalline product was recrystallized from ethyl acetate-ether $(55 \mathrm{ml}: 30 \mathrm{ml})$ to give pure Z-Pro-; $\mathrm{Abu}-\mathrm{OH}(\mathbf{3 1})$ $(0.89 \mathrm{~g}, 53 \%), \operatorname{mp~} 73-75^{\circ} \mathrm{C},[\alpha]_{\mathrm{D}}-29.5^{\circ}\left(c=1, \mathrm{H}_{2} \mathrm{O}\right)$.

Five other protected dipeptides $(33-37)$ were similarly synthesized: Z-Pro- $\gamma \mathrm{Abu}(2 \mathrm{OH})-\mathrm{OH}$ DCHA (33) $(84 \%), \mathrm{mp} 128-130^{\circ} \mathrm{C},[\alpha]_{\mathrm{D}}-18.7^{\circ}(c=1, \mathrm{DMF})$; Z-Phe- $\beta$ Ala-OH (34) $(82 \%), \mathrm{mp} 136-137^{\circ} \mathrm{C},[\alpha]_{\mathrm{D}}-11.5$ $\left(c=1\right.$, DMF); Z-Pro- $\beta$ Ala-OH (35) $(83 \%), \mathrm{mp} 134-136^{\circ} \mathrm{C},[\alpha]_{\mathrm{D}}-31.7^{\circ}(c=1$, DMF); Z-Phe- $\varepsilon$ Acp-OH (36) $(46 \%), \mathrm{mp} 126-128{ }^{\circ} \mathrm{C},[\alpha]_{\mathrm{D}}-10.2 \%\left(c=1\right.$, DMF); Z-Pro- $\varepsilon$ Acp-OH $(37)(92 \%), \operatorname{mp~} 92-94 \mathrm{C},[\alpha]_{\mathrm{D}}-26.2^{\circ}(c=1$, DMF).

b) H-Pro- $\gamma \mathrm{Abu}-\mathrm{OH}(6)$ : Hydrogenation of 31 (4.0 g) was carried out as in method $\mathrm{A} \mathrm{b}$ ) to give pure $\mathrm{H}-\mathrm{Pro}-$ $\gamma \mathrm{Abu}-\mathrm{OH}(6),(1.50 \mathrm{~g}, 63 \%), \mathrm{mp} 202-205^{\circ} \mathrm{C}$ (dec.). Anal. Calcd for $\mathrm{C}_{9} \mathrm{H}_{18} \mathrm{~N}_{2} \mathrm{O}_{3}: \mathrm{C}, 53.98 ; \mathrm{H}, 8.06 ; \mathrm{N}, 13.99$. Found: C, $53.71 ; \mathrm{H}, 8.17 ; \mathrm{N}, 13.66$.

Five other dipeptides $(\mathbf{1 4 - 1 8})$ were similarly obtained: H-Pro- $\gamma \mathrm{Abu}(2 \mathrm{OH})-\mathrm{OH}(\mathbf{1 4})(91 \%), \mathrm{mp} 210-212 \mathrm{C}$. Anal. Calcd for $\mathrm{C}_{9} \mathrm{H}_{16} \mathrm{~N}_{2} \mathrm{O}_{4}$ : C, 49.99; H, 7.46; N, 12.96. Found: C, 50.03; H, 7.70; N, 13.14; H-Phe- $\beta$ Ala-OH (15) $\left(88 \%\right.$ ), mp 218-220 ${ }^{\circ} \mathrm{C}$ (dec.). Anal. Calcd for $\mathrm{C}_{12} \mathrm{H}_{16} \mathrm{~N}_{2} \mathrm{O}_{3}: \mathrm{C}, 61.00 ; \mathrm{H}, 6.83 ; \mathrm{N}, 11.86$. Found: C, 61.13; $\mathrm{H}, 6.93 ; \mathrm{N}$, 11.94; H-Pro- $\beta$ Ala-OH (16) $\left(54 \%\right.$ ), mp 218-219 $\mathrm{C}$ (dec.). Anal. Calcd for $\mathrm{C}_{8} \mathrm{H}_{14} \mathrm{~N}_{2} \mathrm{O}_{3}: \mathrm{C}, 51.60 ; \mathrm{H}, 7.58 ; \mathrm{N}, 15.04$. Found: C, 51.47; H, 7.73; N, 15.08; H-Phe-EAcp-OH (17) $(92 \%)$, mp 130-132 ${ }^{\circ}$ (dec.). Anal. Calcd for

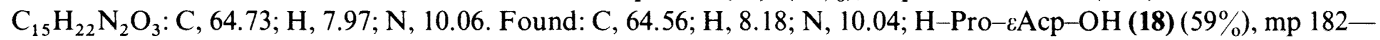
$183^{\circ}$ C. Anal. Calcd for $\mathrm{C}_{11} \mathrm{H}_{20} \mathrm{~N}_{2} \mathrm{O}_{3}: \mathrm{C}, 57.87 ; \mathrm{H}, 8.83 ; \mathrm{N}, 12.27$. Found: C, 58.00; H, 9.09; N, 12.50.

\section{References and Notes}

1) Amino acids, peptides and their derivatives, except Gly, $\beta \mathrm{Ala}, \gamma \mathrm{Abu}, \gamma \mathrm{Abu}(2 \mathrm{OH})$ and $\varepsilon \mathrm{Acp}$, are of the Lconfiguration. Abbreviations used are those recommended by the IUPAC-IUB Commission on Biochemical Nomenclature: Biochemistry, 5, 2485 (1966); ibid., 6, 362 (1967); ibid., 11, 1726 (1972). Z=benzyloxycarbonyl, Boc=tert-butoxycarbonyl, $\quad \mathrm{Bzl}=$ benzyl, $\quad \mathrm{DCC}=N, N^{\prime}$-dicyclohexylcarbodiimide,$\quad \mathrm{HONB}=N$-hydroxy-5norbornene-2,3-dicarboximide, $\mathrm{DSC}=N, N^{\prime}$-disuccinimidyl carbonate, $\mathrm{THF}=$ tetrahydrofuran, $\mathrm{DCU}=$ dicyclohexyl urea, $\mathrm{DMF}=N, N$-dimethylformamide, $\mathrm{TEA}=$ triethylamine.

2) E. Roberts, T. N. Chase and D. B. Tower, "GABA in Nervous System Function," ed. by E. Roberts, T. N. Chase and D. B. Tower, Raven Press, New York, 1976, pp. 1-6.

3) P. L. McGeer and E. G. McGeer, "Basic Neurochemistry," 3rd ed., ed. by G. T. Siegel, R. W. Albers, B. W. Agranoff and R. Katzman, Little Brown and Co., Boston, 1981, pp. 233-253.

4) W. H. Oertel, D. E. Schmechel, E. Mugnaini, M. L. Tappaz and I. J. Kopin, Neuroscience, 6, 2715 (1981).

5) O. P. Ottersen and J. Storm-Mathisen, J. Comp. Neurol., 229, 374 (1984).

6) H. Kimura and T. Tanaka, Neuroscience, in press.

7) B. Onteniente, H. Tago, H. Kimura and T. Maeda, J. Comp. Neurol., 248, 422 (1986).

8) Y. Tomida, H. Kimura, S. Ida and K. Kuriyama, Bull. Jpn. Neurochem. Soc., 24, 193 (1985).

9) A. P. Fosker and H. D. Law, J. Chem. Soc., 1965, 7305.

10) M. Fujino, S. Kobayashi, M. Obayashi, T. Fukuda, S. Shinagawa and O. Nishimura, Chem. Pharm. Bull., 22, 1857 (1974).

11) H. Ogura, T. Kobayashi, K. Shimizu, K. Kawabe and K. Takeda, Tetrahedron Lett., 1979, 4745.

12) S. M. Hsu, L. Raine and H. Fanger, J. Histochem. Cytochem., 29, 557 (1981). 